DIVISION OF THE HUMANITIES AND SOCIAL SCIENCES

CALIFORNIA INSTITUTE OF TECHNOLOGY

PASADENA, CALIFORNIA 91125

EXTERNAL VALIDATION OF VOTER TURNOUT MODELS BY CONCEALED PARAMETER RECOVERY

Antonio Merlo

University of Pennsylvania

Thomas R. Palfrey

California Institute of Technology

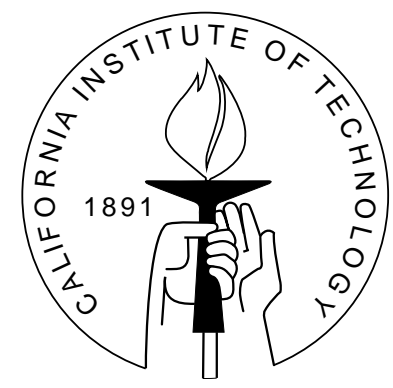

SOCIAL SCIENCE WORKING PAPER 1370

February 2013 


\title{
External validation of voter turnout models by concealed parameter recovery
}

\author{
Antonio Merlo Thomas R. Palfrey
}

\begin{abstract}
We conduct a model validation analysis of several behavioral models of voter turnout, using laboratory data. We call our method of model validation concealed parameter recovery, where estimation of a model is done under a veil of ignorance about some of the experimentally controlled parameters - in this case voting costs. We use quantal response equilibrium as the underlying, common structure for estimation, and estimate models of instrumental voting, altruistic voting, expressive voting, and ethical voting. All the models except the ethical model recover the concealed parameters reasonably well. We also report the results of a counterfactual analysis based on the recovered parameters, to compare the policy implications of the difference models about the cost of a subsidy to increase turnout.
\end{abstract}

We gartefully acknowledge the financial support of the National Science Foundation (SES-0079301, SES-0450712, SES-0094800) and the California Social Science Experimental Laboratory. We thank seminar and conference participants at several institutions for their useful comments and suggestions. Anna Bassi and Claire Lim provided excellent research assistance.

JEL classification numbers: D72, C52, C92

Key words: Turnout, voting, model validation, parameter recovery, laboratory experiments 


\section{Introduction}

For virtually every stylized fact or set of observations in economics and political science there is typically a plethora of alternative models that can provide an explanation for the observed phenomena. Even when imposing the restrictions of the theory on the empirical exercise, it is often the case that several competing structural models can each rationalize the data. How does one validate or compare the empirical performance of structural models? The literature on this topic is vast (see, e.g., Geweke (2007), Geweke and Amisano (2012), Schorfheide and Wolpin (2012) and Wolpin (2007) for surveys of Bayesian and classical methods). Internal validation requires that the model is capable of reproducing the main features of the data used in estimation (within-sample fit). External validation requires that the model can also rationalize aspects of the data that are not used in estimation (out-of-sample fit).

In this paper, we present an external validation analysis of several alternative behavioral models of voter turnout using data from laboratory experiments. Our method of model validation is concealed parameter recovery. In the concealed parameter recovery approach, one carries out structural estimation of a model using actual laboratory data, but under a veil of ignorance about one or more of the experimentally controlled parameters, which we call the concealed parameters. The performance of the model is then assessed with respect to its ability to recover the concealed parameters. We estimate several structural models using the same data and the same estimation procedure (which in our case is maximum likelihood), and then compare their performance with respect to their (quantitative) implications for a counterfactual policy experiment of interest.

We consider several alternative theories of voter turnout that differ with respect to the fundamental mechanism that induces people to vote or to abstain. All these theories (which are described in detail in Section 1.1 below), postulate that voting is costly and individuals are heterogenous with respect to their cost of voting. The calculation of the benefit from voting is, however, different across the different theories and we distinguish between instrumental (or pivotal) voting (where the benefit of voting is based on the extent to which one's

vote may change the outcome of the election), altruistic voting (where the calculation of the benefit of voting also includes the spillover one's voting decision has on other individuals with 
the same preferences over electoral outcomes), expressive voting (where the benefit of voting depends on the utility an individual derives for the sheer act of voting), and ethical voting (where the calculation of the benefit of voting is the solution to a collective problem aimed at maximizing the total welfare of all individuals with the same preferences over electoral outcomes).

For each theory, we specify a structural model which assigns positive probability to all possible levels of turnout (i.e., each structural model has a non degenerate likelihood for all possible data). Following a long-standing tradition in the specification and estimation of discrete-choice models of individual decision making which originated with McFadden's (1981) pathbreaking work, this is done by "augmenting" each model of voter turnout with idiosyncratic choice-specific taste shocks. Since all models are strategic, this leads us to adopt the notion of Quantal Response Equilibrium (QRE) as the natural solution concept in such environments (McKelvey and Palfrey (1995)). Hence, for each behavioral model of voter turnout, the characterization of the QRE generates a likelihood function, which represents the basis for estimation.

We estimate each structural model using a large experimental data set on elections (Levine and Palfrey (2007)). Since the empirical implications of all the theoretical models we consider depend crucially on the distribution of voting costs, which in the experiment is uniform on $[0, C]$, we treat $C$ as the concealed parameter. We then compare the performance of the different estimated models by considering the following policy experiment: Suppose that, for whatever reason, our goal is to increase turnout by 10 percentage points and we want to achieve this goal by implementing a simple policy where each eligible voter receives a cash voucher that can be redeemed only if the individual turns out to vote. How much does the voucher have to be for? Clearly, the answer to this question depends on the estimated structural model one uses to perform the counterfactual policy experiment, which in turn depends crucially on the estimated distribution of voting costs. Our main finding is that the instrumental and the altruistic voting models recover the concealed parameter reasonably well and have similar implications with respect to the policy recommendation they yield. On the other hand, the ethical voting model grossly overestimates the upper bound of the distribution of voting costs, leading to a costly implementation of the "wrong" 
policy. The performance of the expressive voting model is in between.

Our model validation procedure is reminiscent of the Monte Carlo procedure used in econometrics to assess the properties of estimators. In that context, the researcher knows all the parameters of the statistical model which generates the (simulated) data and evaluates the performance of various estimators by their ability to recover the true parameters conditional on knowing the structure of the model. In our context, the researcher knows the parameters of the environment, but does not know the behavioral model(s) which generate the (laboratory) data and evaluates the performance of alternative theories of behavior by their ability to recover the true parameters conditional on assuming, in turn, each structural model as the data generating process and estimating its parameters including the concealed parameters.

Our approach to model validation is similar in spirit to the procedure followed by Bajari and Hortaçsu (2005) who use bid data from auction experiments to assess whether the estimated distribution of valuations that rationalizes the observed bid distribution according to auction theory resembles the actual distribution of valuations assigned to bidders in the experiment. Our work is also somewhat related to a recent literature that uses laboratory data to assess the validity of empirical tests based on field data (Armantier (2002), Frechette et al. (2005), O'Brien and Srivastava (1991), Pezanis-Christou and Santana (2007)). Like our paper, this literature exploits the knowledge of some features of the laboratory environment as the basis for the assessment. ${ }^{1}$

\subsection{Models of Voter Turnout}

The starting point of theoretical research on voter turnout is represented by the "calculus of voting" framework originally formulated by Downs (1957) and later developed by Tullock (1967) and Riker and Ordeshook (1968). According to this framework, given a citizenry of size $N$ facing an election where there are two alternatives (e.g., two candidates or two policy

\footnotetext{
${ }^{1}$ For example, Armantier (2002) runs standard tests that are used in the empirical literature to distinguish between private and common value auction environments using bid data generated from a common value laboratory experiment and shows that they cannot reject the null hypothesis that the data was generated in an auction environment with private values. Frechette et al. (2005) and O'Brien and Srivastava (1991) conduct similar analyses in the context of bargaining environments with different protocols and stock markets with multiple assets, respectively.
} 
proposals), a citizen votes in the election if

$$
p B+d \geq c
$$

and abstains otherwise. Here, $p$ is the probability that the citizen's vote decides the election (i.e., her vote is pivotal), $B$ is the (indirect) benefit to the citizen associated with inducing her desired electoral outcome, $d$ is the (direct) benefit from participating in the election, and $c$ is the citizen's cost of voting in the election. The terms $p B$ and $d$ are often referred to as capturing the instrumental (or investment) and expressive (or consumption) value of voting, respectively.

In the original formulation of the calculus of voting model, $B$ and $d$ are specified as fundamental components of a citizen's preferences and are therefore treated as primitives. Also, $p$ is treated as a parameter which decreases with the size of the electorate. Most of the recent theoretical research on voter turnout has been focused on developing models where $p$, $B$ and $d$ are endogenous variables, derived in equilibrium from more fundamental primitives. Pivotal-voter models (e.g., Borgers (2004), Ledyard (1984) and Palfrey and Rosenthal (1983, 1985)), endogenize the probability that a citizen's vote is decisive, $p$. Altruistic-voter models (e.g., Palfrey and Prisbrey (1996)), provide a theory of the instrumental value of voting, $B$, based on altruism. ${ }^{2}$ Expressive-voter models (e.g., Brennan and Lomasky (1993), Riker and Ordeshook (1968)), provide a foundation for the consumption value of voting, $d$. Ethicalvoter models (e.g., Coate and Conlin (2004), Feddersen and Sandroni (2006) and Harsanyi (1980)), endogenize the concept of civic duty and provide an alternative formulation of both the indirect and the direct benefit from voting. All these models treat the cost of voting, $c$, as exogenous. ${ }^{3}$

The models we specify and estimate are adaptations of these four basic models to a common environment (which is the environment of our experimental data), and to a common

\footnotetext{
${ }^{2}$ Palfrey and Prisbrey (1996) use a weighted altruism model in their estimation of a model of voluntary contributions to public goods. This framework can be easily applied to a voting model and has appeared many places in the experimental and behavioral economics literature.

${ }^{3}$ For models of voter turnout that endogenize the cost of voting see, e.g., Degan and Merlo (2011), Feddersen and Pesendorfer (1996, 1999) and Matsusaka (1995). For a survey of models of voter turnout see, e.g., Merlo (2006).
} 
stochastic framework where individuals receive idiosyncratic, choice-specific taste shocks.

A recent literature has focused on the structural estimation of these models using field data. For example, Coate and Conlin (2004) estimate an ethical-voter model and Coate, Conlin and Moro (2008) estimate a pivotal-voter model and an expressive-voter model using data on local liquor referenda in Texas. ${ }^{4}$ These papers show that while the pivotal-voter model is capable of predicting observed levels of turnout quite well, it also predicts closer electoral outcomes than they are in the data. On the other hand, the ethical-voter model is capable of reproducing most of the features of the data and the empirical performance of the expressive-voter model is in between. As discussed in the Introduction, however, it is not clear how to validate or compare the empirical performance of these structural models. This is especially true given the limitations of field data, and the role played by auxiliary assumptions in the choice of the parametric specifications that are ultimately estimated.

\section{The Voting Environment}

The voting environment we consider is based on Levine and Palfrey (2007), which provided the data for our empirical analysis. There are $N$ voters, divided between two parties: the minority party with $m$ voters; and the majority party with $M$ voters; where $m+M=N$ and $M>m$. There is an election between two candidates, one representing each party. Voting is simultaneous, and each voter faces a binary decision: to vote or to abstain. Voting is costly, and the act of voting has an idiosyncratic cost of $c_{i}$ to voter $i$. Voting costs are private information to each voter, but it is common knowledge that each $c_{i}$ is an independent draw from a distribution of voting costs with support $[0, C]$, and cumulative distribution function $F$. The direct payoff to $i, V_{i}$, depends only on the outcome of the election, whether $i$ votes, and $c_{i}$. There are three possible outcomes: the majority party wins; the minority party wins; or a tie. $W$ denotes the individual benefit to $i$ from his party's candidate winning, which is normalized to 1 . $L$ denotes the individual benefit to $i$ from the other party's candidate winning, which is normalized to 0 . The individual benefit to $i$ of a tie is $(W+L) / 2=0.5$.

\footnotetext{
${ }^{4}$ For an alternative structural estimation of pivotal-voter models using data from local school budget referenda in Oregon, see Hansen, Palfrey and Rosenthal (1987).
} 
Hence, the payoff function is given by:

$$
V_{i}= \begin{cases}1 & \text { if } i^{\prime} \text { s party wins and } i \text { did not vote } \\ 1-c_{i} & \text { if } i^{\prime} \text { s party wins and } i \text { did vote } \\ 0 & \text { if } i^{\prime} \text { s party loses and } i \text { did not vote } \\ -c_{i} & \text { if } i^{\prime} \text { s party loses and } i \text { did vote } \\ 0.5 & \text { if there is a tie and } i \text { did not vote } \\ 0.5-c_{i} & \text { if there is a tie and } i \text { did vote }\end{cases}
$$

\section{Structural Models of Voter Turnout}

In this section, we describe the four structural models of voter turnout we consider and the general procedure we use to implement our concealed parameter recovery approach to assess the performance of these models.

\subsection{Taste Shocks and Quantal Response Equilibrium}

To apply the concealed parameter recovery approach in a uniform fashion to all the different models of voter turnout considered here, we formulate all the models in a similar way. First, each model specifies a set of choices that voters make. In the instrumental voting model, the altruistic voting model, and the expressive voting model, this is simply a binary choice of whether or not to vote. In the ethical voting model, it involves choosing a voting strategy that would be optimal for the group if everyone in the group followed it. We then include an additive taste shock associated with each possible choice in each of the models. The idiosyncratic taste shocks are known to the individual voters when they have to make a decision, but are not directly observable to the econometrician or to the other voters. These shocks produce additive disturbance terms in the payoffs associated to each choice of each player in each voter turnout model. The shocks are assumed to be independent draws from an identical distribution, and the distribution is commonly known to the players in the game. Each player makes a choice, conditional on her taste shocks and cost realization, taking as 
given the probability distribution of choices used by the other voters.

Each model makes equilibrium predictions for observed voting behavior, conditional on parameters of the models and the distribution of taste shocks. That is, the objective functions

of the voters are different than in the original formulation of each model without taste shocks, but behavioral predictions are still disciplined by the equilibrium assumption of rational expectations. Following McFadden (1981), we take the distribution of taste shocks to be specified by an extreme value distribution, leading to choice probabilities that are logit functions. As is now standard in the use of logit models in games, the quantal response component becomes part of the equilibrium (McKelvey and Palfrey $(1995,1998)$ ). Hence, for each model, we characterize its logit quantal response equilibrium (LQRE), which represents the basis for estimation.

\subsection{The Instrumental Voting Model}

In the (pure) model of pivotal voting, or instrumental voting, a voter $i$ turns out to vote if and only if the expected direct benefits to voting exceed the direct costs of voting (see, e.g., Borgers (2004), Ledyard (1984), and Palfrey and Rosenthal (1983, 1985)). The instrumental voting model treats $p$ in equation (1) as an endogenous variable, and takes $B$ and $c$ to be the individual benefits and costs to voting net of idiosyncratic taste shocks, while setting $d=0$. We focus exclusively on quasi-symmetric equilibria, where all voters in the same party use the same strategy. Because we use the logit model of choice, we can separate out the dependence of choice on the private cost and the dependence of choice on the taste shock. With this in mind, we write the behavioral strategy for voter $i$ simply as a mapping $\sigma_{i}: c_{i} \mapsto[0,1]$, which specifies the probability that $i$ votes as a function of $i$ 's private cost realization. Since we are assuming symmetry, equilibrium in the instrumental voting model is characterized by a pair of such functions, $\left(\sigma_{m}^{i n s}, \sigma_{M}^{i n s}\right)$, which specify the voting behavior of a representative member of the minority party and the majority party, respectively.

Given any strategy profile, $\left(\sigma_{m}^{i n s}, \sigma_{M}^{i n s}\right)$, we can directly compute two numbers, $\left(q_{m}^{\text {ins }}, q_{M}^{\text {ins }}\right)$, corresponding the the ex ante probability that a randomly selected voter from party $m$ or 
$M$, respectively, turns out to vote. The calculation is given by:

$$
\begin{aligned}
q_{m}^{i n s} & =\int_{0}^{C} \sigma_{m}^{i n s}(c) d F(c), \\
q_{M}^{i n s} & =\int_{0}^{C} \sigma_{M}^{i n s}(c) d F(c) .
\end{aligned}
$$

These probabilities, in turn, generate $i$ 's pivot probability $p_{i}$, which is the sum of the probability of a tie and the probability the other party is ahead by one vote, if $i$ does not vote. These expressions are the same for all members of a given party, but are slightly different depending on whether $i$ 's party is $m$ or $M$. Denoting these two probabilities by $p_{m}$ and $p_{M}$, the formulas are given by:

$$
\begin{aligned}
& p_{m}= \\
& \sum_{k=0}^{m-1}\left(\begin{array}{c}
m-1 \\
k
\end{array}\right)\left(\begin{array}{c}
M \\
k
\end{array}\right)\left(q_{m}^{i n s}\right)^{k}\left(1-q_{m}^{i n s}\right)^{m-k-1}\left(q_{M}^{\text {ins }}\right)^{k}\left(1-q_{M}^{i n s}\right)^{M-k}+ \\
& \sum_{k=0}^{m-1}\left(\begin{array}{c}
m-1 \\
k
\end{array}\right)\left(\begin{array}{c}
M \\
k+1
\end{array}\right)\left(q_{m}^{i n s}\right)^{k}\left(1-q_{m}^{i n s}\right)^{m-k-1}\left(q_{M}^{i n s}\right)^{k+1}\left(1-q_{M}^{i n s}\right)^{M-k-1},
\end{aligned}
$$

and

$$
\begin{aligned}
& p_{M}= \\
& \sum_{k=0}^{m}\left(\begin{array}{c}
m \\
k
\end{array}\right)\left(\begin{array}{c}
M-1 \\
k
\end{array}\right)\left(q_{m}^{i n s}\right)^{k}\left(1-q_{m}^{i n s}\right)^{m-k}\left(q_{M}^{i n s}\right)^{k}\left(1-q_{M}^{i n s}\right)^{M-k-1}+ \\
& \sum_{k=1}^{m}\left(\begin{array}{c}
m \\
k
\end{array}\right)\left(\begin{array}{c}
M-1 \\
k-1
\end{array}\right)\left(q_{m}^{i n s}\right)^{k}\left(1-q_{m}^{i n s}\right)^{m-k}\left(q_{M}^{i n s}\right)^{k-1}\left(1-q_{M}^{i n s}\right)^{M-k}
\end{aligned}
$$

In the logit quantal response equilibrium of the instrumental voting model, the log odds ratio of choosing to vote rather than not vote is proportional to the difference between the expected direct payoff of voting, net of voting cost, and the expected direct payoff of abstaining. Simple calculations show that this difference in expected payoffs equals $c-\frac{1}{2} p_{m}$ for a member of the minority party and $c-\frac{1}{2} p_{M}$ for a voter in the majority party. The proportionality factor equals the precision of the distribution of taste shocks, denoted by $\lambda$. Therefore, a voter's turnout probability is a continuous and strictly decreasing probability 
function of her voting cost, which is exactly equal to .5 at the indifference point between voting and abstaining. The exact formulas are given by the logit response functions:

$$
\begin{aligned}
\sigma_{m}^{i n s}(c) & =\frac{1}{1+e^{\lambda\left(c-\frac{1}{2} p_{m}\right)}}, \\
\sigma_{M}^{i n s}(c) & =\frac{1}{1+e^{\lambda\left(c-\frac{1}{2} p_{M}\right)}},
\end{aligned}
$$

with $p_{m}$ and $p_{M}$ defined in equations (5) and (6).

A logit quantal response equilibrium (LQRE) for the instrumental voting model is a pair of turnout functions, $\sigma^{i n s}=\left(\sigma_{m}^{i n s}, \sigma_{M}^{i n s}\right)$, satisfying equations $(3),(4),(5),(6),(7),(8)$.

\subsection{The Altruistic Voting Model}

The (group) altruistic model of voting extends the basic model of preferences by letting individuals value the payoffs of other members of their group, in addition to their private payoff from voting (see, e.g., Palfrey and Prisbrey (1996)). In this model, the utility to voter $i, U_{i}$, is given by a weighted average of $i$ 's own direct payoff and the total direct payoff to $i$ 's group:

$$
U_{i}=\left\{\begin{array}{cc}
(1-\alpha) V_{i}+\alpha V_{m} & \text { if } i \in m \\
(1-\alpha) V_{i}+\alpha V_{M} & \text { if } i \in M
\end{array}\right.
$$

where the total group payoffs are given by:

$$
\begin{aligned}
& V_{m}=\sum_{i \in m} V_{i} \\
& V_{M}=\sum_{i \in M} V_{i} .
\end{aligned}
$$

Thus, the altruistic voting model also treats $p$ in equation (1) as an endogenous variable and $c$ as an individual cost of voting, as in the instrumental voting model, but now $B$ takes into account not only the personal benefits of changing the outcome of an election, but also the benefits that accrue to all the other members of a voter's group. ${ }^{5}$

\footnotetext{
${ }^{5}$ In this model, it is still the case that $d=0$. In the special case of $\alpha=0$ for all $i$, the model reduces to the pure model of instrumental voting described in the previous subsection.
} 
The equilibrium characterization for the altruistic voting model is similar to the one for the instrumental voting model, except for the different structure of the payoffs. In particular, the logit choice equations for an $m$-voter and an $M$-voter are slightly different because the utility effects of an individual voting or abstaining are magnified by her altruism parameter. Specifically:

$$
\begin{aligned}
& \sigma_{m}^{a l t}(c ; \alpha)=\frac{1}{1+e^{\lambda\left(c-\frac{1}{2}(1-\alpha+\alpha m) p_{m}\right)}} \\
& \sigma_{M}^{a l t}(c ; \alpha)=\frac{1}{1+e^{\lambda\left(c-\frac{1}{2}(1-\alpha+\alpha M) p_{M}\right)}} .
\end{aligned}
$$

The logit constant, $\lambda$, is again the response parameter that ranges from 0 to $\infty$. The pivot probabilities, $p_{m}$ and $p_{M}$, are derived from $q_{m}^{\text {alt }}$ and $q_{M}^{\text {alt }}$ similarly to (5) and (6), where:

$$
\begin{aligned}
q_{m}^{\text {alt }} & =\int_{0}^{C} \sigma_{m}^{\text {alt }}(c ; \alpha) d F(c), \\
q_{M}^{\text {alt }} & =\int_{0}^{C} \sigma_{M}^{\text {alt }}(c ; \alpha) d F(c) .
\end{aligned}
$$

A logit quantal response equilibrium (LQRE) for the (group) altruistic model of voting with altruism coefficient $\alpha$ is a pair of turnout functions, $\sigma^{\text {alt }}=\left(\sigma_{m}^{\text {alt }}, \sigma_{M}^{a l t}\right)$, satisfying equations (5), (6), (10), (11), (12), and (13), substituting $q_{m}^{\text {alt }}$ and $q_{M}^{\text {alt }}$ for $q_{m}^{\text {ins }}$ and $q_{M}^{\text {ins }}$ in equations (5) and (6).

\subsection{The Expressive Voting model}

The expressive model of voting is based on the idea that voters turn out because they get direct utility from publicly expressing their preferences (see, e.g., Brennan and Lomasky (1993)). ${ }^{6}$ In this model, a voter does not compare the expected benefit of voting to $c$, but compares it to the "net" voting cost $c-d$, where $d$ is an additive expressive voting benefit leading to a reduction in the voting cost for unspecified reasons (such as, for example, citizen duty). ${ }^{7}$

\footnotetext{
${ }^{6}$ In the context of the experimental environments we study here, this model is equivalent to what Riker and Ordeshook (1968) first put forth formally as the "citizen duty" model.

${ }^{7}$ Citizen duty and preference for expression are only two of the many possible rationales for the inclusion of a $d$-term in the calculus of voting equation that have been put forth over the years. For example, Tullock
} 
The pure expressive voting model is a non-equilibrium model of voter turnout, in the sense that the $p$ and $B$ terms in equation (1) play no role at all: A voter receives a utility of $d$ from voting, suffers a cost $c$ from voting, and turns out to vote if and only if $d>c$. Since the predictions of this model do not depend on any of the features of the voting environment like, for example, $N, m$, or $M$, the pure model of expressive voting is immediately rejected by the data. ${ }^{8}$ Hence, we consider a modified model of expressive voting which adds an expressive component, $d$, to the pure model of instrumental voting described above.

Specifically, the logit response functions for an $m$-voter and an $M$-voter, which characterize the voting behavior of a representative member of the minority party and the majority party, respectively, are given by:

$$
\begin{aligned}
\sigma_{m}^{e x}(c ; d) & =\frac{1}{1+e^{\lambda\left(c-d-\frac{1}{2} p_{m}\right)}}, \\
\sigma_{M}^{e x}(c ; d) & =\frac{1}{1+e^{\lambda\left(c-d-\frac{1}{2} p_{M}\right)}},
\end{aligned}
$$

and the pivot probabilities, $p_{m}$ and $p_{M}$, are derived from $q_{m}^{e x}$ and $q_{M}^{e x}$ similarly to (5) and (6), where:

$$
\begin{aligned}
q_{m}^{e x} & =\int_{0}^{C} \sigma_{m}^{e x}(c ; d) d F(c), \\
q_{M}^{e x} & =\int_{0}^{C} \sigma_{M}^{e x}(c ; d) d F(c) .
\end{aligned}
$$

A logit quantal response equilibrium (LQRE) for the expressive model of voting with expressive coefficient $d$ is a pair of turnout functions, $\sigma^{e x}=\left(\sigma_{m}^{e x}, \sigma_{M}^{e x}\right)$, satisfying equations (5), (6), (14), (15), (16), and (17), substituting $q_{m}^{e x}$ and $q_{M}^{e x}$ for $q_{m}^{i n s}$ and $q_{M}^{i n s}$ in equations (5) and $(6)$.

\subsection{The Ethical Voting Model}

The (group) ethical model of voting relies on a calculus of voting that is very different from the individual calculations of the previous models. The general idea of ethical models is (1967) observed that this net cost of voting could just as easily be negative as positive.

${ }^{8}$ In particular, the turnout rates predicted by the model are constant across elections and across groups. 
that there is a "right" strategy for a voter to adopt, in the sense that if everyone in a group adopted this strategy, then an efficient outcome for the group would arise (see, e.g., Feddersen and Sandroni (2006) and Harsanyi (1980)). For example, in the prisoner's dilemma game, the right action is to cooperate, because, if everyone cooperated, it would maximize the total payoff to the two players. The subtlety here is that the right action depends not only on the underlying parameters of the game, but the efficiency calculation is only applied relative to the "right outcome", which differs depending on whether the voter belongs to the majority or the minority party.

The model of ethical voting we consider follows closely the "group-ethical" model proposed by Coate and Conlin (2004). ${ }^{9}$ In this model, the ethical choice is defined by the strategy that would maximize the expected total benefits to the members of one's own party, assuming all voters in that party used this strategy, taking as given the strategy used by all voters of the other party (who are also ethical, but with respect to a different group). ${ }^{10}$ However, unlike Coate and Conlin, we consider a voting environment with a finite number of voters and we modify the payoffs to include additive taste shocks. ${ }^{11}$

The derivation of the equilibrium is somewhat different than in the models described in the previous subsections. In particular, one can think of it as resulting from the optimization problems faced by two social planners (one for each party), who are behaving strategically with each other. For clarity, we first introduce the model without any taste shocks. All voters are assumed to be ethical, and the decision problem is viewed by an ethical voter as the solution to a two person game between their party and the other party. An ethical $m$-voter calculates a critical cost level $c_{m}^{e t h}$ to maximize the expected total payoff of the voters in the minority party, given the expected turnout rates in the $M$-party. The critical cost level specifies a group cutoff rule whereby a member of the minority party votes if and only if $c \leq c_{m}^{e t h}$. The payoff to an ethical $m$-voter resulting from a particular cutoff level is the

\footnotetext{
${ }^{9}$ This is also similar to earlier models of turnout and public good provision studied by Morton (1991).

${ }^{10}$ Without this "group utilitarian" modification, the solution would be trivial: One voter from the majority party should vote, while everyone else abstains.

${ }^{11}$ Coate and Conlin also assume that $m$ and $M$ are random variables, which is necessary for them to generate randomness in the electoral outcomes in equilibrium. We do not do this here for two reasons. First, in the experiments that generated the data, $m$ and $M$ are common knowledge. Second, because we have a finite number of voters, the outcomes of elections are random in equilibrium even if $m$ and $M$ are common knowledge.
} 
expected total payoff to the members in the $m$ party, if all members adopt that cutoff rule:

$$
V_{m}\left(c_{m}^{e t h} \mid q_{M}^{e t h}\right)=m\left(W_{m}\left(c_{m}^{e t h}, q_{M}^{e t h}\right)-\int_{0}^{c_{m}^{e t h}} c d F(c)\right)
$$

where $W_{m}$ is the probability that the minority candidate wins plus $\frac{1}{2}$ times the probability of a tie, and $q_{M}^{e t h}$ is the probability a randomly selected $M$-voter votes in the group ethical equilibrium. The second term in equation (18) is the expected cost incurred by a member of the group, using a cutoff level of $c_{m}^{e t h}$. The exact expression for $W_{m}$ is:

$$
\begin{aligned}
W_{m}\left(c_{m}^{e t h}, q_{M}^{\text {eth }}\right)= & \\
& \frac{1}{2} \sum_{k=0}^{m}\left(\begin{array}{c}
m \\
k
\end{array}\right)\left(\begin{array}{c}
M \\
k
\end{array}\right)\left(q_{M}^{\text {eth }}\right)^{k}\left(1-q_{M}^{\text {eth }}\right)^{M-k}\left(F\left(c_{m}^{\text {eth }}\right)\right)^{k}\left(1-F\left(c_{m}^{\text {eth }}\right)\right)^{m-k}+ \\
& \sum_{k=1}^{m} \sum_{j=0}^{k-1}\left(\begin{array}{c}
m \\
k
\end{array}\right)\left(\begin{array}{c}
M \\
j
\end{array}\right)\left(q_{M}^{\text {eth }}\right)^{j}\left(1-q_{M}^{\text {eth }}\right)^{M-j}\left(F\left(c_{m}^{\text {eth }}\right)\right)^{k}\left(1-F\left(c_{m}^{\text {eth }}\right)\right)^{m-k} .
\end{aligned}
$$

Similarly, an ethical $M$-voter chooses $c_{M}^{e t h}$ to maximize the expected total payoff of the voters in the majority party, given the expected turnout rates in the $m$-party:

$$
V_{M}\left(c_{M}^{e t h} \mid q_{m}^{e t h}\right)=M\left(W_{M}\left(c_{M}^{e t h}, q_{m}^{e t h}\right)-\int_{0}^{c_{M}^{e t h}} c d F(c)\right)
$$

where $W_{M}$ is the probability that the majority candidate wins plus $\frac{1}{2}$ times the probability of a tie. This is given by:

$$
\begin{aligned}
W_{M}\left(c_{M}^{\text {eth }}, q_{m}^{\text {eth }}\right)= & \\
& \frac{1}{2} \sum_{k=0}^{m}\left(\begin{array}{c}
m \\
k
\end{array}\right)\left(\begin{array}{c}
M \\
k
\end{array}\right)\left(q_{m}^{\text {eth }}\right)^{k}\left(1-q_{m}^{\text {eth }}\right)^{m-k}\left(F\left(c_{M}^{\text {eth }}\right)\right)^{k}\left(1-F\left(c_{M}^{\text {eth }}\right)\right)^{M-k}+ \\
& \sum_{k=0}^{m} \sum_{j=k+1}^{M}\left(\begin{array}{c}
m \\
k
\end{array}\right)\left(\begin{array}{c}
M \\
j
\end{array}\right)\left(q_{m}^{\text {eth }}\right)^{k}\left(1-q_{m}^{\text {eth }}\right)^{m-k}\left(F\left(c_{M}^{\text {eth }}\right)\right)^{j}\left(1-F\left(c_{M}^{\text {eth }}\right)\right)^{M-j} .
\end{aligned}
$$

The way we introduce taste shocks into the model is to assume that a voter's selection of the ethical rule follows a logit function. That is, rather than choosing the optimal rule with probability one, an ethical $m$-voter ( $M$-voter) chooses a rule in proportion to its exponen- 
tiated expected group payoff net of costs, taking as given the turnout probability of voters from the other party. This specification is consistent with viewing the "choice" of an ethical voter as a cutoff rule, so that the (choice-specific) taste shocks are just additive terms to $V_{m}$ or $V_{M}$, depending on the voter's party. ${ }^{12}$ Thus, the probabilities an $m$-voter chooses $c_{m}^{e t h}$ and an $M$-voter chooses $c_{M}^{e t h}$ are given by:

$$
\begin{aligned}
\pi_{m}\left(c_{m}^{e t h}\right) & =\frac{e^{\lambda m\left(W_{m}\left(c_{m}^{e t h}, q_{M}^{e t h}\right)-\int_{0}^{c_{m}^{e t h}} c d F(c)\right)}}{\int_{0}^{C} e^{\lambda m\left(W_{m}\left(x, q_{M}^{e t h}\right)-\int_{0}^{x} c d F(c)\right)} d x}, \\
\pi_{M}\left(c_{M}^{e t h}\right) & =\frac{e^{\lambda M\left(W_{M}\left(c_{M}^{e t h}, q_{m}^{e t h}\right)-\int_{0}^{c_{M}^{e t h}} c d F(c)\right)}}{\int_{0}^{C} e^{\lambda M\left(W_{M}\left(x, q_{m}^{e t h}\right)-\int_{0}^{x} c d F(c)\right)} d x} .
\end{aligned}
$$

The formulas for $\sigma_{m}^{e t h}$ and $\sigma_{M}^{e t h}$ (the implied cost-conditional vote probabilities for an ethical $m$-voter and an ethical $M$-voter, respectively), are then given by the probability that the voter chooses a cutpoint $x \geq c$. That is:

$$
\begin{aligned}
\sigma_{m}^{e t h}(c) & =\int_{c}^{C} \pi_{m}(x) d x \\
\sigma_{M}^{e t h}(c) & =\int_{c}^{C} \pi_{M}(x) d x .
\end{aligned}
$$

Taking expectations with respect to $c$, we obtain:

$$
\begin{aligned}
q_{m}^{e t h} & =\int_{0}^{C} \sigma_{m}^{e t h}(c) d F(c), \\
q_{M}^{e t h} & =\int_{0}^{C} \sigma_{M}^{e t h}(c) d F(c) .
\end{aligned}
$$

A logit quantal response equilibrium (LQRE) for the (group) ethical model of voting is a pair of turnout functions, $\sigma^{\text {eth }}=\left(\sigma_{m}^{e t h}, \sigma_{M}^{e t h}\right)$, satisfying equations (19), (21), (22), (23), (24), (25), (26), and (27).

\footnotetext{
${ }^{12}$ Note that, in this case, the choice set is continuous, while in the previous models it is discrete.
} 


\section{The Data}

The data set we use in our analysis was taken from Levine and Palfrey (2007), and contains 1,500 laboratory elections conducted with incentivized human subjects in the CASSEL laboratory at UCLA. In each election, $N$ subjects were randomly divided into two parties of size $m$ and $M$, with $0<m<M<N$. Each voter was independently assigned a voting cost for that election, $c_{i}$, from a uniform distribution on the interval $[0, .55]$. So $F=$ Uniform and $C=.55$ in all elections in our data set. All of this was common knowledge among the participants of the election. However, each voter's voting cost and party assignment was private information. All voters made independent decisions simultaneously to either vote for their party's candidate, or abstain. After everyone voted, the number of votes cast for each party was reported to each participant, and payoffs accrued. The experimental design had six different treatments, where $N, m$, and $M$ were systematically varied. The experimental design is summarized in Table $1 .^{13}$

Table 1: Data Summary

\begin{tabular}{|c|c|c|c|c|c|c|c|}
\hline \hline Treatment & $N$ & $m$ & $M$ & \# of elections & $m$-turnout & $M$-turnout & Overall Turnout \\
\hline \hline 1 & 9 & 3 & 6 & 450 & 0.44 & 0.40 & 0.41 \\
2 & 9 & 4 & 5 & 450 & 0.48 & 0.45 & 0.47 \\
3 & 27 & 9 & 18 & 200 & 0.38 & 0.28 & 0.31 \\
4 & 27 & 13 & 14 & 200 & 0.39 & 0.36 & 0.37 \\
5 & 51 & 17 & 34 & 100 & 0.33 & 0.27 & 0.29 \\
6 & 51 & 25 & 26 & 100 & 0.39 & 0.36 & 0.38 \\
\hline \hline
\end{tabular}

Thus, our data set contains 30,900 individual-level observations (4,950 in treatment 1, 4,950 in treatment 2, 5,400 in treatment 3, 5,400 in treatment 4, 5,100 in treatment 5, and 5,100 in treatment 6): 9,900 for 9-voter elections, 10,800 for 27-voter elections and 10,200 for 51-voter elections. The last three columns of Table 1 report the observed turnout rates for

\footnotetext{
${ }^{13}$ The Levine and Palfrey data also contain 850 elections with 3 voters (i.e., a treatment with $N=3$, $m=1$, and $M=2$ ). We do not include these elections in our analysis since, when there are only three voters, the notion of group, which plays a critical role in the altruistic and the ethical model, is not applicable to the minority party (and is also not very meaningful for the majority party).
} 
the two parties in each of the treatments as well as the overall turnout in each treatment. ${ }^{14}$

\section{Results}

Using the data described in the previous section, we estimate each of the four structural models of voter turnout described in Section 3 by maximum likelihood. ${ }^{15}$ In the estimation, we ignore the actual costs drawn by the voters in each election, and we treat the upper bound of the distribution of voting costs as the concealed parameter of interest. ${ }^{16}$ Recall that in the actual experiment, the distribution of voting costs $F(c)$ was uniform on $[0, .55]$. Hence, we estimate $C$ under the maintained hypothesis that the cost distribution is uniform on $[0, C]$. For each model, we also estimate $\lambda$, the precision parameter of the extreme value distribution of taste shocks. In addition, for the altruistic model and the expressive model we also estimate the altruism parameter $\alpha$ and the expressive parameter $d$, respectively.

Table 2 reports the parameter estimates, their standard errors and the maximized value of the log-likelihood function for each of the four models. ${ }^{17}$

Table 2: Estimation Results*

\begin{tabular}{|c|c|c|c|c|}
\hline \hline Parameter & Instrumental & Altruistic & Expressive & Ethical \\
\hline \hline$C$ & $0.573(0.017)$ & $0.590(0.021)$ & $0.837(0.295)$ & $5.904(0.220)$ \\
$\lambda$ & $4.054(0.251)$ & $3.961(0.265)$ & $4.773(1.203)$ & $0.112(0.010)$ \\
$\alpha$ & & $0.004(0.004)$ & & \\
$d$ & & & $0.132(0.146)$ & \\
\hline \hline Log-likelihood & -20132.13 & -20131.84 & -20132.01 & -20150.64 \\
\hline \hline
\end{tabular}

* Standard errors are in parentheses.

\footnotetext{
${ }^{14}$ For further details about the experimental protocol, design, and results, see Levine and Palfrey (2007).

${ }^{15}$ While we do not have a general uniqueness proof of the LQRE for the four voting models we consider, we do know that the Bayesian equilibrium (without QRE disturbances) in each of these models is unique for the experimental environment considered here. For this environment, our numerical procedure for computing the LQRE equilibria for each of the four models never encountered issues related to multiplicity for all possible parameter values.

${ }^{16}$ This is different from the QRE estimation in Levine and Palfrey (2007), which conditioned estimation of a pivotal-voter model on all of the experimental parameters as well as the realized idiosyncratic costs.

${ }^{17}$ Note that the estimated value of $\lambda$ in the ethical model is not comparable to the ones in the three other models since the choice set in the ethical model is continuous (voters choose cutoff rules), while in the other models it is discrete (voters choose whether to vote or to abstain).
} 
For the concealed parameter recovery method of model validation to have maximum power, it would be ideal to have a "level playing field" for all the models. In other words, ideally, we would want the various models to fit the data equally well, using standard measures of (within-sample) fit. Table 3 reports predicted turnout rates for each of the four models and compares them to the turnout rates observed in the data.

Table 3: Predicted and Observed Turnout Rates

\begin{tabular}{|c|c|c|c|c|c|}
\hline \hline Elections & Instrumental & Altruistic & Expressive & Ethical & Data \\
\hline \hline$N=9$ & 0.44 & 0.44 & 0.44 & 0.44 & 0.44 \\
$N=27$ & 0.35 & 0.35 & 0.35 & 0.36 & 0.34 \\
$N=51$ & 0.32 & 0.32 & 0.32 & 0.33 & 0.33 \\
\hline \hline All Elections & 0.37 & 0.37 & 0.37 & 0.37 & 0.37 \\
\hline \hline
\end{tabular}

As we can see form Table 3, each model is capable of reproducing the turnout rates observed in the data equally well. Hence, none of the models can be rejected based on standard within-sample goodness-of-fit tests. We therefore turn our attention to the performance of the models with respect to their ability of recovering the concealed parameter $C$.

For each model, we conduct a simple concealed-parameter-recovery hypothesis test by asking the following question: Is the estimated distribution of costs significantly different from Uniform on $[0, .55]$ ? The answer is clearly yes for the ethical model. The estimated upper bound of 5.9 is more than twenty four standard errors away from .55. The group ethical model fails to recover anything close to the true distribution of costs for the simple reason that it predicts extremely high turnout in most of the experimental treatments. In order to fit the data, the model must estimate the distribution of voting costs to be an order of magnitude higher than they actually were. Thus, the concealed parameter recovery procedure clearly rejects the group ethical model.

The other three models all produce similar estimates of $C$ that are in the ballpark of the true value of $.55 .^{18}$ In fact, for the instrumental, the altruistic and the expressive model

\footnotetext{
${ }^{18}$ Note that the instrumental model is nested in both the altruistic and the expressive models (corresponding to $\alpha=0$ and $d=0$, respectively). Neither $\alpha$ nor $d$ are estimated to be significantly different from 0 at any conventional significance level, and a likelihood ratio test fails to reject the constrained model (instrumental) in favor of either of the two models with an additional parameter.
} 
the estimated upper bound of the cost distribution is within two standard errors of the true value. However, the point estimate of $C$ for the expressive model is considerably larger than the estimates for the instrumental and altruistic model, and its standard error is also much larger. ${ }^{19}$ We conclude that the concealed parameter recovery procedure cannot reject the instrumental and altruistic models. Also, the performance of the expressive model with respect to the concealed parameter recovery criterion is in between those of the instrumental and altruistic models and that of the ethical model.

The key remaining question is whether the ability or inability of a model to successfully recover the concealed parameter of interest has any practical implication that is substantively important. To address this question, we compare the policy implications of the four different models of voter turnout we estimated, by performing a counterfactual policy experiment that directly affects the cost of voting.

The use of structural models is often advocated on the grounds that the estimated model can be used to conduct counterfactual policy experiments. Here, we estimated four different structural models using the same data and showed that they all fit the data well. However, policy recommendations based on these models can be very different. There are many possible policy experiments related to voter turnout that could be considered. One common complaint about elections is that too small a fraction of eligible voters turn out to vote in major elections. Some democratic theorists have even argued that significant voter participation is a necessary condition for the viability of democratic systems. A simple policy to raise turnout, which is consistent with any of the four theoretical models considered here, would be to reduce the cost of voting. For example, the government could give a subsidy to any voter who turns out to vote. ${ }^{20}$ However, depending on which model the government uses to guide its choice of policy, the potential cost of such policy for any given target in terms of overall turnout could vary dramatically.

Suppose, for example, that the government wished to raise turnout by 10 percentage points by issuing a cash voucher to any voter who turns out to vote. In the context of the

\footnotetext{
${ }^{19}$ The reason why both $C$ and $d$ are imprecisely estimated in the expressive model is that separate identification of the two parameters is tenuous.

${ }^{20}$ Equivalently a fine could be imposed on non-voters. Many countries have compulsory voting that is enforced with fines (e.g., Australia).
} 
voting environment we consider, how much would such a policy cost according to each of the four models we estimated?

The effect of a subsidy $S$, is simply to shift the support of the cost distribution from $[0, C]$ to $[-S, C-S]$. There are two ways to compute how large $S$ has to be for each model. One simple way, which is naive and ignores game-theoretic considerations, is to simply shift costs down by $10 \%$ of the maximum voting cost. Because the estimate of the maximum voting cost varies across the models, this naive (or back-of-the-envelope) calculation gives different answers depending on the model. The second, more sophisticated way, is to take into consideration what each model predicts to be the equilibrium effect of a shift in the distribution of voting costs on voter turnout, and compute how large $S$ has to be to achieve a 10 percentage point increase in turnout. In the context of our data, that would mean an increase in turnout from 0.37 to 0.47 . This equilibrium calculation requires computing the equilibrium of the model for different values of $S$, using the estimated parameters of each model. If $C_{j}$ is model $j$ 's estimate of the upper bound on the cost distribution (where $j=\{$ Instrumental, Altruistic, Expressive, Ethical $\}$ ), then we compute the equilibrium turnout of that model for a distribution of costs uniform on $\left[-S, C_{j}-S\right]$ for different values of $S$ until we find the value of $S$ that would theoretically produce a $47 \%$ overall turnout in our elections. Such a subsidy represents the policy recommendation based on that model to achieve a 10 percentage points increase in turnout.

Table 4 shows how much this voting subsidy would have to be for each of the four models, computed both according to the naive calculation as well as the equilibrium calculation.

Table 4: Voting Subsidy

\begin{tabular}{|c|c|c|}
\hline \hline Models & Naive Calculation & Equilibrium Calculation \\
\hline \hline Instrumental & 0.06 & 0.13 \\
Altruistic & 0.06 & 0.13 \\
Expressive & 0.08 & 0.13 \\
Ethical & 0.59 & 1.43 \\
\hline \hline
\end{tabular}

According to the equilibrium calculations reported in Table 4, the instrumental, altruistic and expressive models all recommend a relatively small subsidy, which would shift the 
distribution of voting costs down by about $25 \%$ of $C(S=.13$ for all three models $) .{ }^{21}$ In contrast, the policy recommendation based on the estimated ethical model entails a much larger subsidy, equal to 1.43 according to the equilibrium calculation. ${ }^{22}$ This is eleven times the subsidy recommended by the other three models to achieve the same target. In fact, shifting the cost distribution down by 1.43 would produce a cost distribution that is uniform on $[-1.43,-.88]$, so the actual result of implementing the policy would be to yield nearly $100 \%$ turnout according to any of the four models. ${ }^{23}$ This policy grossly misses the target of increasing turnout by 10 percentage points, and would be extremely costly. Even worse, it would be extremely inefficient, since approximately the same level of turnout could obviously be achieved at a much lower cost. ${ }^{24}$ The concealed parameter recovery method of model validation would prevent the "wrong" policy from being implemented, since the ethical model would be rejected.

\section{Conclusions}

The concealed parameter recovery method we have proposed in this paper as a way to validate structural models can be applied to many other contexts in addition to models of voter turnout. $^{25}$ Also, the basic insights of this method can be extended to non-laboratory environments, provided there are fixed characteristics of the data that are accurately measured and can be treated as "concealed variables". Further progress and broader application of this methodological approach will ultimately require the theoretical development of a formal test and a characterization of its statistical properties. This is especially important in situations where the recovery entails multiple concealed parameters. We intend to pursue this avenue of research in future work.

\footnotetext{
${ }^{21}$ Note that this is about twice the subsidy based on the naive calculation corresponding to shifting the voting cost distribution down by $10 \%$ of $C$ ( $S=.06$ or .08 , depending on the model).

${ }^{22}$ The subsidy is equal to .64 according to the naive calculation.

${ }^{23}$ It is "nearly" rather than "exactly" because all the models have a QRE component, so even voters with negative (net) voting costs abstain with positive probability. The same would be true if we were to shift the cost distribution down by .64 according to the naive calculation.

${ }^{24}$ As long as $S>.55$, all voters would have negative net voting costs and hence turnout would be nearly $100 \%$.

${ }^{25}$ Obviously, it can also be applied to assess the empirical performance of many other models of voter turnout in addition to the ones considered here.
} 


\section{References}

[1] Armantier, O. (2002). "Deciding Between the Common and Private Values Paradigm: An Application to Experimental Data," International Economic Review, 43: 783-801.

[2] Bajari, P. and A. Hortaçsu (2005). "Are Structural Estimates of Auction Models Reasonable? Evidence from Experimental Data," Journal of Political Economy, 113: 703-41.

[3] Borgers, T. (2004). "Costly Voting," American Economic Review, 94: 57-66.

[4] Brennan, G. and L. Lomasky (1993). Democracy and Decision: The Pure Theory of Electoral Preference. Cambridge: Cambridge University Press.

[5] Coate, S. and M. Conlin (2004). "A Group Rule-Utilitarian Approach to Voter Turnout: Theory and Evidence." American Economic Review, 94: 1476-1504.

[6] Coate, S. M. Conlin, and A. Moro (2008). "The Performance of Pivotal-Voter Models in Small-Scale Elections: Evidence from Texas Liquor Referenda," Journal of Public Economics, 92: 582-96.

[7] Degan, A. and A. Merlo (2011). "A Structural Model of Turnout and Voting in Multiple Elections," Journal of the European Economic Association, 9: 1-37.

[8] Downs, A. (1957). An Economic Theory of Democracy. New York: Harper and Row.

[9] Feddersen, T. and A. Sandroni (2006). "A Theory of Participation in Elections," American Economic Review, 96: 1271-82.

[10] Feddersen, T. and W. Pesendorfer (1996). "The Swing Voter's Curse," American Economic Review, 86: 408-24.

[11] Feddersen, T. and W. Pesendorfer (1999). "Abstention in Elections with Asymmetric Information and Diverse Preferences," American Political Science Review, 93: 381-98.

[12] Frechette, G., J. Kagel, and M. Morelli (2005). "Behavioral Identification in Coalitional Bargaining: An Experimental Analysis of Demand Bargaining and Alternating Offers," Econometrica, 73: 1893-1937. 
[13] Geweke, J. (2007). "Bayesian Model Comparison and Validation," American Economic Review, Papers and Proceedings, 97: 60-4.

[14] Geweke, J. and G. Amisano (2012). "Prediction with Misspecified Models," American Economic Review, Papers and Proceedings, 102: 482-486.

[15] Hansen, S., T. Palfrey, and H. Rosenthal (1987). "The Downsian Model of Electoral Participation: Formal Theory and Empirical Analysis of the Constituency Size Effect," Public Choice, 52: 15-33.

[16] Harsanyi, J. (1980). "Rule Utilitarianism, Rights, Obligations, and the Theory of Rational Behavior," Theory and Decision, 12: 115-33.

[17] Ledyard, J. (1984). "The Pure Theory of Large Two-Candidate Elections," Public Choice, 44: 7-41.

[18] Levine, D. and T. Palfrey (2007) "The Paradox of Voter Participation? A Laboratory Study," American Political Science Review, 101: 143-58.

[19] McFadden, D. (1981). "Econometric Models of Probabilistic Choice" in C. Manski and D. McFadden (eds.) Structural Analysis of Discrete Data with Econometric Applications, Cambridge, MA: MIT Press.

[20] McKelvey, R. and T. Palfrey (1995). "Quantal Response Equilibria in Normal Form Games," Games and Economic Behavior, 10: 6-38.

[21] McKelvey, R. and T. Palfrey (1998). "Quantal Response Equilibria in Extensive Form Games," Experimental Economics, 1: 9-41.

[22] Matsusaka, J. (1995). "Explaining Voter Turnout Patterns: An Information Theory," Public Choice, 84: 91-117.

[23] Merlo, A. (2006). "Whither Political Economy? Theories, Facts and Issues," in Richard Blundell, Whitney Newey and Torsten Persson (eds.), Advances in Economics and Econometrics, Theory and Applications: Ninth World Congress of the Econometric Society, Cambridge: Cambridge University Press. 
[24] Morton, R. (1991). "Groups in Rational Turnout Models," American Journal of Political Science, 35: 758-76.

[25] O'Brien, J. and S. Srivastava (1991). "Dynamic Stock Markets with Multiple Assets: An Experimental Analysis," Journal of Finance, 46: 1811-38.

[26] Palfrey, T. and J. Prisbrey (1997). "Anomalous Behavior in Linear Public Goods Experiments: How Much and Why?" American Economic Review, 87: 829-46.

[27] Palfrey, T. and H. Rosenthal (1983). "A Strategic Calculus of Voting," Public Choice, 41: 7-53.

[28] Palfrey, T. and H. Rosenthal (1985). "Voter Participation and Strategic Uncertainty," American Political Science Review, 79: 62-78.

[29] Pezanis-Christou, P. and A. Romeu Santana (2007). "Structural Inferences from FirstPrice Auction Data," Working Paper.

[30] Riker, W. and P. Ordeshook (1968). "A Theory of the Calculus of Voting," American Political Science Review, 62: 25-42.

[31] Schorfheide, F. and K. Wolpin (2012). "On the Use of Holdout Samples for Model Selection," American Economic Review, Papers and Proceedings, 102: 477-81.

[32] Tullock, G. (1968). Toward a Mathematics of Politics. Ann Arbor, MI: University of Michigan Press.

[33] Wolpin, K. (2007). "Ex Ante Policy Evaluation, Structural Estimation, and Model Selection," American Economic Review, Papers and Proceedings, 97: 48-52. 